\title{
Aortic Pulse Wave Velocity in Peritoneal Dialysis Patients Is Not Simply Associated with Extracellular Water Expansion
}

\author{
Kamonwan Tangvoraphonkchai ${ }^{a}$ Andrew Davenport $^{\mathrm{b}}$ \\ ${ }^{a}$ Faculty of Medicine, Mahasarakham University, Mahasarakham, Thailand; b UCL Centre for \\ Nephrology, Royal Free Hospital, University College London, London, UK
}

\section{Keywords}

Hypertension · Peritoneal dialysis · Bioimpedance $\cdot$ Icodextrin $\cdot$ Pulse wave velocity

\begin{abstract}
Background: Cardiac death is increased in peritoneal dialysis (PD) patients. Pulse wave velocity (PWV) is a measurement of arterial stiffness, and previous reports linked PWV to increased extracellular water (ECW). As cyclers and icodextrin are increasingly used, we wished to determine whether this association between PWV and ECW remains. Methods: We measured aortic PWV (aPWV) and bioimpedance (InBody, Seoul, South Korea) in consecutive PD patients attending for peritoneal membrane testing. Results: 189 patients were included, $62.4 \%$ male, mean age $63.1 \pm 15.2$ years, $45.3 \%$ diabetic, median dialysis duration 12.3 (6.5-25.1) months, $71.4 \%$ using cyclers, weight $73.0 \pm 16.1 \mathrm{~kg}$, systolic blood pressure $142 \pm 21 \mathrm{~mm} \mathrm{Hg}$, aPWV $10.4 \pm 5.1 \mathrm{~m} / \mathrm{s}$. aPWV was associated with pulse pressure $(r=0.26, p=0.001)$, Davies comorbidity score $(r=0.18, p=0.013)$, and $\mathrm{N}$-terminal pro-brain-type natriuretic peptide (NTproBNP; $r=0.18, p=0.011)$. Patients with aPWV $\geq 10 \mathrm{~m} / \mathrm{s}$ were older $(65.9 \pm 13.6$ vs. $60.1 \pm$ 16.3 years, $p<0.01)$ with a higher ECW-to-total body water ratio $(0.400 \pm 0.012$ vs. $0.396 \pm$ $0.013, p<0.05)$, but ECW/height was not different ( $8.52 \pm 2.32 \mathrm{vs} .8 .75 \pm 1.78 \mathrm{~L} / \mathrm{m})$, as was NTproBNP $(2,472[788-5,422]$ vs. 1,234 [410-6,230] ng/L). On multivariable testing, aPWV was positively associated with $\beta$-blocker prescription (standardised $\beta$ coefficient [St $\beta$ ] 0.3, 95\% confidence limits [95\% CL] 0.7-2.6, $p=0.001$ ) and negatively with icodextrin prescription (St $\beta$ $0.19,95 \% C L-0.2$ to $-2.1, p=0.04)$. Conclusions: Compared to previous studies, we did not find an independent association between $\mathrm{PPWV}$ and ECW and estimates of ECW excess, using the InBody bioimpedance device, suggesting that vascular stiffness in PD patients is more complex than simple ECW volume expansion in PD patients.




\section{Kidney \\ Blood Pressure \\ Research}

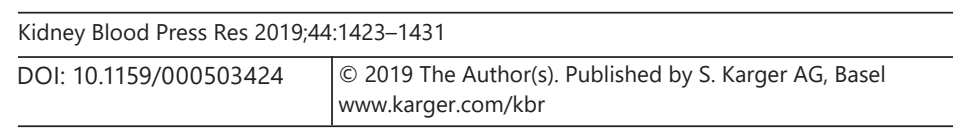

Tangvoraphonkchai and Davenport: Pulse Wave Velocity in Peritoneal Dialysis Patients

\section{Background}

Although there has been some improvement in the survival of dialysis patients in recent times, mortality remains high, compared to the general population, with 5-year survival rates similar to or worse than those of some of the more common solid organ malignancies [1]. Cardiovascular causes continue to be the major contributor of this excess mortality. Whereas atheromatous coronary artery disease with cholesterol-rich plaque formation is the predominant cardiovascular risk factor for the general population, dialysis patients typically develop arteriosclerosis leading to increased risk of heart failure, stroke and cardiac arrhythmias [2].

Arterial stiffness is one of the first clinically detectable indicators of arteriosclerosis. Pulse wave velocity (PWV) is the most validated non-invasive method of determining arterial stiffness, and as such is considered the gold standard measurement [3, 4]. Aortic stiffness is recognised as an important independent risk factor for cardiovascular mortality in the general population and also for dialysis patients [5]. Previous studies of PWV measurements in peritoneal dialysis (PD) patients have varied in their observations, with some suggesting an association with both lower serum albumin and residual renal function and higher C-reactive protein (CRP) [6-8], whereas others have reported associations with extracellular water (ECW) overhydration [9-11]. Due to these differences in the reported literature, and changes in patient demographics and PD prescriptions over time, we decided to prospectively measure PWV in a cohort of PD patients, to determine whether patients with an increased ECW had higher PWV.

\section{Patients and Methods}

The electronic health care records of 189 adult patients with chronic kidney disease stage 5 , who had been treated with PD and had PWV measurements taken when attending for peritoneal membrane assessment, were reviewed. Peritoneal transport was calculated as the 4-h peritoneal dialysate effluent creatinine-to-serum ratio (peritoneal equilibrium test), weekly urea clearance $\left(\mathrm{Kt} / \mathrm{V}_{\text {urea }}\right)$ and dietary normalised protein nitrogen appearance (nPNA) calculated by standard methods from 24-h urine and peritoneal dialysate effluent samples [12]. No patient had been treated for PD peritonitis or had an acute hospital admission within the preceding 3 months. All patients had been on PD for a minimum of 2 months.

We used an 8-electrode multi-frequency segmental bioimpedance device (InBody 720, Seoul, South Korea) following a standardised protocol, making measurements after patients had passed urine and the peritoneal dialysate had been drained out $[13,14]$. Measurements were made on the same day as the peritoneal equilibrium test and aortic PWV (aPWV). We measured total body water (TBW), ECW, intracellular water (ICW) and body composition in the standing position. The bioimpedance device was regularly serviced and calibrated. We made estimates of increased ECW as the ratio of ECW/TBW, ECW/height and ECW/ICW and body weight [15]. Blood tests were taken concurrently and analysed by standard methods (Roche Integra, Roche diagnostics, Lewes, UK) for urea, creatinine, albumin, haemoglobin, $\mathrm{CRP}$, haemoglobin $\mathrm{A}_{1 \mathrm{c}}\left(\mathrm{HbA}_{1 \mathrm{c}}\right)$ and $\mathrm{N}$-terminal pro-brain-type natriuretic peptide (NTproBNP) $[16,17]$. Patient comorbidity was assessed using the Davies comorbidity scoring system [18].

Aortic-brachial PWV was measured using the Tensio Clinic Ateriograph (TensioMed Kft., Budapest, Hungary) which has been validated against direct invasive measurements [19] and has also been shown to be the most reproducible of the currently available devices for measuring PWV [20]. In keeping with standard practice, aPWV measurements were corrected for heart rate. PWV measurements were not able to be recorded in patients with atrial fibrillation, other arrhythmias, and patients with no recordable upper arm blood pressure 
Table 1. Patient demographics and results of peritoneal membrane assessment in peritoneal dialysis (PD) patients with aortic pulse wave velocity (PWV) $\leq 10 \mathrm{~cm} / \mathrm{s}$ and $>10 \mathrm{~cm} / \mathrm{s}$

\begin{tabular}{|c|c|c|}
\hline Variable & $\mathrm{PWV} \leq 10 \mathrm{~m} / \mathrm{s}$ & $\mathrm{PWV}>10 \mathrm{~m} / \mathrm{s}$ \\
\hline Number & 92 & 97 \\
\hline Male, $\%$ & 60.9 & 63.9 \\
\hline Diabetic, \% & 31.9 & 51.5 \\
\hline White ethnicity, \% & 43.5 & 41.2 \\
\hline Age, years & $60.1 \pm 16.3$ & $65.9 \pm 13.6^{* *}$ \\
\hline Weight, kg & $72.7 \pm 16.6$ & $73.7 \pm 16.0$ \\
\hline Current smoker, \% & 9.8 & 6.2 \\
\hline Ex-smoker, \% & 37.0 & 45.4 \\
\hline Davies comorbidity score & $1(0-2)$ & $1(1-2)^{*}$ \\
\hline New York Heart Association grade & $2(1-2)$ & $2(1-3)^{*}$ \\
\hline ACEI/ARB prescription, $\%$ & 22.8 & $10.3^{*}$ \\
\hline Beta-blocker prescription, $\%$ & 44.6 & $66.0^{* *}$ \\
\hline Antihypertensives prescribed, \% & 77.2 & 83.5 \\
\hline Statin prescription, $\%$ & 65.2 & 69.1 \\
\hline Mean arterial blood pressure, $\mathrm{mm} \mathrm{Hg}$ & $102.0 \pm 14.7$ & $100.1 \pm 13.8$ \\
\hline PD duration, months & $12.0(8.0-21.5)$ & $10.0(4.0-22.0)$ \\
\hline CAPD, $\%$ & 33.7 & 23.7 \\
\hline APD dry day, \% & 9.8 & 21.6 \\
\hline APD wet day, $\%$ & 56.5 & 54.6 \\
\hline Icodextrin usage, $\%$ & 88.0 & $74.2^{*}$ \\
\hline $22.7 \%$ dextrose usage, $\%$ & 47.8 & 54.6 \\
\hline Icodextrin, L/day & $1.5(1.0-2.0)$ & $1.4(0-2.0)$ \\
\hline $22.7 \%$ dextrose L/day & $0(0-4.7)$ & $3.9(0-4.8)$ \\
\hline 4-h dialysate/serum creatinine & $0.76 \pm 0.11$ & $0.74 \pm 0.12$ \\
\hline Weekly urine Kt/ $\mathrm{U}_{\text {urea }}$ & $0.89(0.33-0.89)$ & $0.84(0.38-1.3)$ \\
\hline Weekly peritoneal Kt/ $\mathrm{V}_{\text {urea }}$ & $1.22(0.92-1.48)$ & $1.16(0.95-1.44)$ \\
\hline Total weekly Kt/ $\mathrm{V}_{\text {urea }}$ & $2.0(1.62-2.57)$ & $2.06(1.65-2.42)$ \\
\hline 24-h urine volume, L/day & $0.73(0.39-1.30)$ & $0.82(0.34-1.25)$ \\
\hline Protein nitrogen appearance rate, g/day & $64.3 \pm 17.3$ & $61.4 \pm 15.1$ \\
\hline
\end{tabular}

ACEI, angiotensin-converting enzyme inhibitor; APD, automated peritoneal dialysis; ARB, angiotensin receptor blocker; CAPD, continuous ambulatory peritoneal dialysis. Results expressed as integer, percentage, mean $\pm \mathrm{SD}$, median (interquartile range). ${ }^{*} p<0.05,{ }^{* *} p<0.01$.

recordings. We used the European Society of Hypertension consensus of a PWV of $>10 \mathrm{~m} / \mathrm{s}$ to reflect abnormal PWV [21].

Patients underwent clinical examination at the time of PWV and were graded by the same clinical observer according to whether the jugular venous pulse was elevated and peripheral oedema graded on a 4-point scale (0: no oedema on finger compression over the lower tibia, 1: minimal oedema - minor indentation but no visible pitting, 2 : some oedema - visible pitting after compression, 3: moderate oedema - oedema visible on inspection and pitting greater than grade 2).

Two-dimensional echocardiograms were performed (Philips IE33, Philips Medical Systems, Eindhoven, the Netherlands) in a subset of 169 (89.4\%) patients. Diastolic dysfunction was graded in keeping with the American Society of Echocardiography guidelines [22, 23].

\section{Statistical Analysis}

Data were checked for normality using the D'Agostino and Pearson normality test, and results were expressed as mean \pm standard deviation, median and interquartile range, or 
Table 2. Peritoneal dialysis patient bioimpedance body composition and biochemical results for those with aortic pulse wave velocity (PWV) $\leq 10 \mathrm{~cm} / \mathrm{s}$ and $>10 \mathrm{~cm} / \mathrm{s}$

\begin{tabular}{lll}
\hline Variable & PWV $\leq 10 \mathrm{~m} / \mathrm{s}$ & PWV $>10 \mathrm{~m} / \mathrm{s}$ \\
\hline Skeletal muscle mass, kg & $27.2 \pm 7.1$ & $26.7 \pm 6.2$ \\
Fat mass, \% & $29.7 \pm 11.7$ & $32.0 \pm 9.8$ \\
Visceral fat area, cm ${ }^{2}$ & $109.8 \pm 47.7$ & $124.9 \pm 48.9^{*}$ \\
Intracellular water, L & $22.4 \pm 5.5$ & $22.0 \pm 4.8$ \\
Extracellular water, L & $14.4 \pm 3.7$ & $14.7 \pm 3.3$ \\
ECW/TBW & $0.396 \pm 0.013$ & $0.400 \pm 0.012^{*}$ \\
ECW/height, L/m & $8.52 \pm 2.32$ & $8.75 \pm 1.78$ \\
Overhydration, L & $0.92(0.32-1.2)$ & $0.96(0.67-1.72)$ \\
Haemoglobin, g/L & $109.5 \pm 15.9$ & $111.8 \pm 15.3$ \\
\hline Serum albumin, g/L & $37.7 \pm 4.6$ & $37.9 \pm 4.2$ \\
Serum sodium, mmol/L & $136.1 \pm 4.3$ & $136.8 \pm 5.0$ \\
Serum cholesterol, mmol/L & $4.7 \pm 1.3$ & $4.5 \pm 1.2$ \\
Serum LDL cholesterol, mmol/L & $2.4 \pm 1.1$ & $2.3 \pm 1.0$ \\
Serum urea, mmol/L & $20.6 \pm 6.4$ & $18.9 \pm 5.6 *$ \\
Serum creatinine, $\mu$ mol/L & $690(522-870)$ & $608(494-806)$ \\
Serum C-reactive protein, mg/L & $4.5(2.0-9.0)$ & $3.5(2.0-11.0)$ \\
NTproBNP, ng/L & $1,234(410-6,230)$ & $2,472(788-5,422)$ \\
Serum ferritin, $\mu$ g/L & $650(315-962)$ & $555(355-879)$ \\
\hline
\end{tabular}

ECW/TBW, extracellular water/total body water; LDL, low-density cholesterol; NTproBNP, serum $\mathrm{N}$-terminal pro-brain-type natriuretic peptide. Results expressed as integer, percentage, mean $\pm \mathrm{SD}$, median (interquartile range). ${ }^{*} p<0.05$.

percentage. The Student $t$, Mann-Whitney $\mathrm{U}$ and $\chi^{2}$ tests, with corrections where appropriate, were used to compare groups, and non-parametric data were log transformed if required for further analysis. Factors individually associated with aPWV were determined using a multivariable regression model, using variables which correlated with PWV $(p<0.1)$ by univariate analysis. The model was checked for collinearity. Statistical analysis was performed using Graph Pad Prism (version 8.0, Graph Pad, San Diego, CA, USA) and SPSS (version 24, IBM, Armonk, New York, NY, USA). Statistical significance was taken at or below the 5\% level.

\section{Results}

aPWV was measured in 189 patients by the same observer; 118 (62.4\%) were male, mean age was $63.1 \pm 15.2$ years, $86(45.3 \%)$ were diabetic, with median dialysis vintage of 12.3 (6.5-25.1) months. Their mean weight was $73.0 \pm 16.1 \mathrm{~kg}$, systolic blood pressure was $142 \pm 21 \mathrm{~mm} \mathrm{Hg}$, diastolic blood pressure was $81 \pm 14 \mathrm{~mm} \mathrm{Hg}$, PWV was $10.5 \pm 4.1 \mathrm{~m} / \mathrm{s}$ and heart rate-adjusted PWV was $10.4 \pm 5.1 \mathrm{~m} / \mathrm{s}$. Only 1 patient had an elevated jugular venous pulse, $55 \%$ of the patients had no lower limb oedema, $33.9 \%$ had minimal oedema, $10.6 \%$ had some oedema, and $1.1 \%$ observable oedema.

We divided the patients into those with a lower aPWV of $\leq 10$ and a higher aPWV of $>10$ $\mathrm{cm} / \mathrm{s}$ [21] (Table 1). Patients with greater aPWV were older and fewer used icodextrin dialysates. More patients with higher aPWV were prescribed beta-blockers, but fewerwere prescribed angiotensin-converting enzyme inhibitors and angiotensin receptor blockers (ARBs). However, mean arterial blood pressure did not differ between the groups (Table 1). There was no difference in body composition between the groups apart from visceral fat (Table 2), but those patients 
Table 3. Peritoneal dialysis patient blood pressure and echocardiography results for those with aortic pulse wave velocity ( $\mathrm{PWV}$ ) $\leq 10 \mathrm{~cm} / \mathrm{s}$ and $>10 \mathrm{~cm} / \mathrm{s}$

\begin{tabular}{lll}
\hline Variable & PWV $\leq 10 \mathrm{~m} / \mathrm{s}$ & PWV $>10 \mathrm{~m} / \mathrm{s}$ \\
\hline LV ejection fraction, \% & $52.7 \pm 10.3$ & $49.5 \pm 12.9$ \\
LV mass index, g/m ${ }^{2}$ & $148.1 \pm 53.0$ & $146.6 \pm 47.6$ \\
LV hypertrophy, \% & 44.6 & 41.2 \\
LV diastolic dysfunction grade & $0.70 \pm 0.59$ & $0.98 \pm 0.74^{*}$ \\
AV peak gradient, mm Hg & $7.4 \pm 3.6$ & $10.5 \pm 7.9^{*}$ \\
Aortic PWV, m/s & $7.99 \pm 1.53$ & $12.12 \pm 2.05^{* * *}$ \\
Aortic pulse pressure, mm Hg & $62.6 \pm 19.7$ & $76.4 \pm 19.9^{* * *}$ \\
Aortic SBP, mm Hg & $163.0 \pm 30.9$ & $171.4 \pm 27.1^{*}$ \\
Return time, s & $54.0 \pm 27$ & $95.2 \pm 16.3^{* * *}$ \\
Brachial augmentation index & $-5.8(-36.9$ to 16.0$)$ & $16.3(-2.3 \text { to } 37.7)^{* * *}$ \\
Aortic augmentation index & $31.2(15.3$ to 41.3$)$ & $45.7(36.5 \text { to } 54.0)^{* * *}$ \\
No peripheral oedema, \% & 56.5 & 53.6 \\
\hline
\end{tabular}

LV, left ventricle; AV, aortic valve; SBP, systolic blood pressure. Results expressed as integer, percentage, mean $\pm \mathrm{SD}$, median (interquartile range). ${ }^{*} p<0.05,{ }^{* *} p<0.01,{ }^{* * *} p<0.001$.

Table 4. Multivariable regression model of aortic pulse wave velocity

\begin{tabular}{lllllll}
\hline Variable & $\beta$ & StE $\beta$ & St $\beta$ & $t$ & $95 \%$ CL & $p$ \\
\hline BB & 1.67 & 0.47 & 0.30 & 3.6 & 0.7 to 2.6 & 0.001 \\
Icodextrin & -1.26 & 0.60 & -0.19 & -2.1 & -2.5 to -0.1 & 0.036 \\
Log CRP & -1.17 & 0.60 & -0.22 & -2.0 & -2.3 to -0.01 & 0.049 \\
\hline
\end{tabular}

Adjusted for age, gender, use of $22.7 \%$ dextrose, icodextrin, serum urea, albumin, C-reactive protein (CRP), N-terminal brain natriuretic peptide, ferritin, Davies comorbidity score, New York Heart Association grade (NYHA), prescription of beta-blockers (BB), angiotensin-converting enzyme inhibitors or angiotensinconverting enzyme inhibitors, statins, smoking status, left ventricular mass index, glycosylated haemoglobin, percentage body fat. If appropriate non-parametric variables were log converted. Standard error of $\beta$ (StE $\beta$ ), standardised $\beta$ (St $\beta), 95 \%$ confidence limits (95\% CL). $R^{2}=0.22$.

with higher aPWV had an increased ECW/TBW ratio and lower serum urea concentrations. On echocardiography, left ventricular mass and left ventricular mass adjusted for body size [23] were not different (Table 3). Excluding measurements associated with vascular stiffness, echocardiographic assessments of the peak aortic valve gradient and left ventricular diastolic dysfunction were greater for those with higher aPWV. NTproBNP concentrations were not different between the groups. Although there were no significant differences in the clinical examination of patients with regard to signs of fluid excess, patients with greater aPWV had higher New York Heart Association (NYHA) grades of heart failure.

On univariate analysis, PWV was associated with pulse pressure $(r=0.26, p=0.001)$, Davies comorbidity score $(r=0.18, p=0.013)$, NTproBNP $(r=0.18, p=0.011)$, and negatively with nPNA $(r=-1.07, p=0.024)$. A number of other variables had weaker positive associations, $0.05<p<0.1$, including age, ECW/TBW, glycated haemoglobin, urea, litres of dialysate used/day, ECW overhydration, left ventricular ejection fraction, and ferritin. A multivariable regression model showed that aPWV was associated with greater prescription of beta-blockers, but less usage of icodextrin dialysates and lower log CRP values (Table 4). 


\section{Kidney \\ Blood Pressure \\ Research}

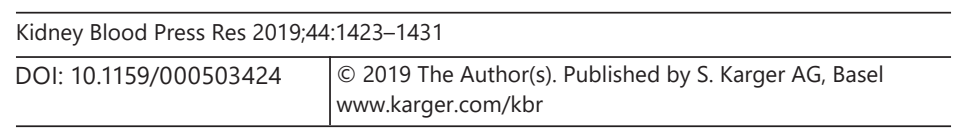

Tangvoraphonkchai and Davenport: Pulse Wave Velocity in Peritoneal Dialysis Patients

\section{Discussion}

PWV is currently considered the "gold" standard for the non-invasive measurement of arterial stiffness, due to the reliability of measurements $[3,4]$. We measured aPWV, as this is more reproducible compared to carotid-femoral measurements, which combine both central and conduit arteries [19, 24].

In the general population, PWV increases with age [25], and those in our cohort with an aPWV $>10 \mathrm{~m} / \mathrm{s}$ were older. Previous studies in PD patients have reported a variable effect of age, with some studies reporting an association [26, 27] and others no association [10,11]. As expected, there was an association between aPWV and pulse pressure, and more patients with a higher aPWV had diastolic dysfunction on echocardiography. Several studies have reported an association between PWV and increased ECW overhydration $[9,10]$. There has been a debate as to the relative importance of ECW volume expansion and hypertension in dialysis patients, since, although the majority of patients with ECW expansion have hypertension, volume status does not explain all cases $[28,29]$. Although our patients with a higher aPWV had a greater ECW/ TBW ratio, there was no difference when adjusting ECW for height, or when calculating overhydration based on the difference between measured ECW and that expected based on measured ICW [15]. Whereas most previous studies in PD patients have used various bioimpedance machines (Xitron technology, San Diego, CA, USA) [16], and bioimpedance measurements have been made in the supine position with peritoneal dialysate in situ, we used a different device for measurements made with the peritoneal dialysate drained out and in the standing position. Bioimpedance machines use different algorithms, and as such may not be directly comparable. We have previously studied both devices [30]. Bioimpedance measurements can be affected by the presence of intra-abdominal fluid [31], and if not, enough time has been allowed for fluid to equilibrate on taking the supine position [32]. We have previously demonstrated an association between NTproBNP and increased ECW in PD patients [33], and in this study, NTproBNP was not significantly different between the normal and high aPWV groups.

We were unable to demonstrate any significant association between aPWV and left ventricular mass. This may be due to differences in the quality and duration of pre-dialysis medical care and the introduction of clinical guideline targets for blood pressure control [34], and other studies measuring a composite of central and conduit artery PWV $[9,10]$. However, more importantly, most of these previous studies failed to report on the clinical examination of patients as to whether patients had clinical signs of ECW expansion or symptomatic heart failure $[9,10]$.

As PWV is a marker of vascular stiffness, the association with prescription of beta-blocker antihypertensives is not unexpected. More patients prescribed angiotensin-converting enzyme inhibitors and ARBs had lower aPWV, and this effect was most marked with ARBs alone $\left(\chi^{2}=5.0, p=0.025\right)$. This finding is in keeping with a previous report suggesting that ARBs, particularly when combined with HMGCoA3 reductase inhibitors (statins), are associated with lower PWV [35].

Some studies have reported an effect of body size on PWV [11] but we did not find such an association, although there was more visceral fat in our patients with higher aPWV, which has been previously reported, and there is a recognised association between visceral adiposity and cardiovascular mortality [36].

Our study differs from previous reports in that the majority of our patients used icodextrin compared to earlier studies [9, 10, 26, 27], and icodextrin has been shown to improve hydration status and reduce ECW expansion compared to standard and higher glucosecontaining dialysates [37]. As such, the lower aPWV in patients prescribed icodextrin would support an association between ECW and aPWV, but in our study, only 1 patient had clinical signs of ECW overhydration with a raised jugular venous pulse and peripheral oedema. 


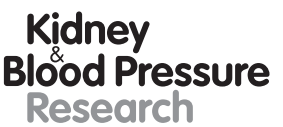

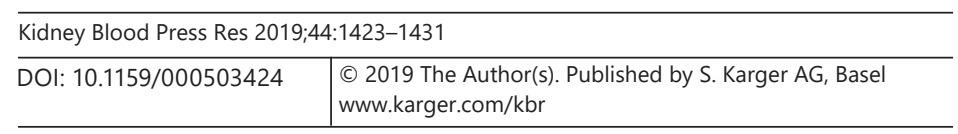

Tangvoraphonkchai and Davenport: Pulse Wave Velocity in Peritoneal Dialysis Patients

Previous studies have reported an association between inflammation and PWV $[8,26$, $38]$, and inflammatory states can lead to an increase in ECW $[16,17]$. However, we noted the opposite in that patients with higher CRP values were associated with lower aPWV in our multivariable model, although equally other studies have reported no effect of inflammatory cytokines on PWV [39]. We used a single measurement of CRP taken on the day of testing and not a time-averaged measurement, and so the CRP may not have been representative, and minor changes may have affected aPWV. As with any cross-sectional study, we can only report associations rather than causality, and similarly without a longitudinal follow-up we cannot comment on survival, although in the UK PD technique and patient survival is confounded by the relatively high rates of change in modalities, both to transplantation and haemodialysis. Our study differs from previous reports due to the number of patients treated by overnight cyclers compared to continuous ambulatory PD and the high usage of icodextrin. In addition, compared to other studies, we measured aPWV and ECW in the erect position with dialysate drained out with a multifrequency device.

Compared to previous studies, we did not find an independent association between faster aPWV and ECW and between faster aPWV and estimates of ECW excess, suggesting that vascular stiffness in PD patients is more complex than simply due to ECW volume expansion alone in PD patients [40], as we found an association with beta-blockers, despite adjusting for heart rate, and lower aPWV in patients using icodextrin, which may result in greater sodium losses [41], and markers of inflammation, such as CRP.

\section{Statement of Ethics}

Study approval was granted by the UK NHS national ethics committee.

IRAS project number 129559; the study was undertaken in keeping with the Helsinki accord with appropriate informed consent and trial registration (ISRCTN70556765).

\section{Disclosure Statement}

The authors have no conflict of interest. The data in this paper have not been previously presented or published. K.T. was in receipt of an International Society of Nephrology training fellowship award.

\section{Funding Sources}

The authors received no funding.

\section{Author Contributions}

A.D. obtained ethical approval. K.T. made measurements of PWV. K.T. and A.D. analysed data. K.T. and A.D. wrote the paper. 
Tangvoraphonkchai and Davenport: Pulse Wave Velocity in Peritoneal Dialysis Patients

\section{References}

1 Nordio M, Limido A, Maggiore U, Nichelatti M, Postorino M, Quintaliani G; Italian Dialysis and Transplantation Registry. Survival in patients treated by long-term dialysis compared with the general population. Am J Kidney Dis. 2012 Jun;59(6):819-28.

2 Seliger SL, Gillen DL, Tirschwell D, Wasse H, Kestenbaum BR, Stehman-Breen CO. Risk factors for incident stroke among patients with end-stage renal disease. J Am Soc Nephrol. 2003 Oct;14(10):2623-31.

3 Cavalcante JL, Lima JA, Redheuil A, Al-Mallah MH. Aortic stiffness: current understanding and future directions. J Am Coll Cardiol. 2011 Apr; 57(14):1511-22.

4 Sigrist MK, Chiarelli G, Levin A, Romann A, Weber C. Pulse wave velocity measurements are reproducible in multiple trained observers: a short report. Nephron Clin Pract. 2010;116(1):c60-4.

5 Covic A, Gusbeth-Tatomir P, Goldsmith DJ. Arterial stiffness in renal patients: an update. Am J Kidney Dis. 2005 Jun;45(6):965-77.

6 Kuang DW, Li CL, Kuok UI, Cheung K, Lio WI, Xin J. Risk factors associated with brachial-ankle pulse wave velocity among peritoneal dialysis patients in Macao. BMC Nephrol. 2012 Nov 1;13:143.

7 Huang WH, Chen KH, Hsu CW, Chen YC, Hung CC, Huang JY, et al. Residual renal function - one of the factors associated with arterial stiffness in peritoneal dialysis patients. Insight from a retrospective study in 146 peritoneal dialysis patients. Blood Purif. 2008;26(2):133-7.

8 Stompór T, Rajzer M, Sułowicz W, Dembińska-Kieć A, Janda K, Kawecka-Jaszcz K, et al. An association between aortic pulse wave velocity, blood pressure and chronic inflammation in ESRD patients on peritoneal dialysis. Int J Artif Organs. 2003 Mar;26(3):188-95.

9 Akdam H, Öğünç H, Alp A, Özbek Ö, Ömürlü İK, Yeniçerioğlu Y, et al. Assessment of volume status and arterial stiffness in chronic kidney disease. Ren Fail. 2014 Feb;36(1):28-34.

10 Kocyigit I, Sipahioglu MH, Orscelik O, Unal A, Celik A, Abbas SR, et al. The association between arterial stiffness and fluid status in peritoneal dialysis patients. Perit Dial Int. 2014 Nov-Dec;34(7):781-90.

11 Kwan BC, Szeto CC, Chow KM, Law MC, Cheng MS, Leung CB, et al. Bioimpedance spectroscopy for the detection of fluid overload in Chinese peritoneal dialysis patients. Perit Dial Int. 2014 Jun;34(4):409-16.

12 NKF-DOQI clinical practice guidelines for peritoneal dialysis adequacy. Assessment of Nutritional status. Am J Kidney Dis. 2007;30(3 Suppl 2):S125-9.

13 Davenport A. Does peritoneal dialysate affect body composition assessments using multi-frequency bioimpedance in peritoneal dialysis patients? Eur J Clin Nutr. 2013 Feb;67(2):223-5.

14 Fürstenberg A, Davenport A. Assessment of body composition in peritoneal dialysis patients using bioelectrical impedance and dual-energy x-ray absorptiometry. Am J Nephrol. 2011;33(2):150-6.

15 Yoowannakul S, Kotecha T, Fontana M, Davenport A. Do Pre-Hemodialysis Estimates of Extracellular Volume Excess Using Bioimpedance and N-Terminal Brain Natriuretic Peptide Correlate With Cardiac Chamber Size Measured by Magnetic Resonance Imaging? Ther Apher Dial. 2019 Aug;23(4):362-68.

16 Papakrivopoulou E, Lillywhite S, Davenport A. Is N-terminal probrain-type natriuretic peptide a clinically useful biomarker of volume overload in peritoneal dialysis patients? Nephrol Dial Transplant. 2012 Jan;27(1):396-401.

17 Booth J, Pinney J, Davenport A. N-terminal proBNP-marker of cardiac dysfunction, fluid overload, or malnutrition in hemodialysis patients? Clin J Am Soc Nephrol. 2010 Jun;5(6):1036-40.

18 Davies SJ, Phillips L, Naish PF, Russell GI. Quantifying comorbidity in peritoneal dialysis patients and its relationship to other predictors of survival. Nephrol Dial Transplant. 2002 Jun;17(6):1085-92.

19 Horváth IG, Németh A, Lenkey Z, Alessandri N, Tufano F, Kis P, et al. Invasive validation of a new oscillometric device (Arteriograph) for measuring augmentation index, central blood pressure and aortic pulse wave velocity. J Hypertens. 2010 Oct;28(10):2068-75.

20 Davies JM, Bailey MA, Griffin KJ, Scott DJ. Pulse wave velocity and the non-invasive methods used to assess it: Complior, SphygmoCor, Arteriograph and Vicorder. Vascular. 2012 Dec;20(6):342-9.

21 Van Bortel LM, Laurent S, Boutouyrie P, Chowienczyk P, Cruickshank JK, De Backer T, et al.; Artery Society; European Society of Hypertension Working Group on Vascular Structure and Function; European Network for Noninvasive Investigation of Large Arteries. Expert consensus document on the measurement of aortic stiffness in daily practice using carotid-femoral pulse wave velocity. J Hypertens. 2012 Mar;30(3):445-8.

22 Nagueh SF, Smiseth OA, Appleton CP, Byrd BF 3rd, Dokainish H, Edvardsen T, et al.; Houston, Texas; Oslo, Norway; Phoenix, Arizona; Nashville, Tennessee; Hamilton, Ontario, Canada; Uppsala, Sweden; Ghent and Liège, Belgium; Cleveland, Ohio; Novara, Italy; Rochester, Minnesota; Bucharest, Romania; and St. Louis, Missouri. Recommendations for the Evaluation of Left Ventricular Diastolic Function by Echocardiography: An Update from the American Society of Echocardiography and the European Association of Cardiovascular Imaging. Eur Heart J Cardiovasc Imaging. 2016 Dec;17(12):1321-60.

23 Lang RM, Bierig M, Devereux RB, Flachskampf FA, Foster E, Pellikka PA, et al.; American Society of Echocardiography's Nomenclature and Standards Committee; Task Force on Chamber Quantification; American College of Cardiology Echocardiography Committee; American Heart Association; European Association of Echocardiography, European Society of Cardiology. Recommendations for chamber quantification. Eur J Echocardiogr. 2006 Mar;7(2):79-108.

24 Nemcsik J, Egresits J, El Hadj Othmane T, Fekete BC, Fodor E, Szabó T, et al. Validation of arteriograph - a new oscillometric device to measure arterial stiffness in patients on maintenance hemodialysis. Kidney Blood Press Res. 2009;32(3):223-9. 
25 Reference Values for Arterial Stiffness' Collaboration. Determinants of pulse wave velocity in healthy people and in the presence of cardiovascular risk factors: 'establishing normal and reference values'. Eur Heart J. 2010 Oct;31(19):2338-50.

26 Cheng LT, Tang LJ, Chen HM, Tang W, Wang T. Relationship between serum albumin and pulse wave velocity in patients on continuous ambulatory peritoneal dialysis. Vasc Health Risk Manag. 2008;4(4):871-6.

27 Gao N, Kwan BC, Chow KM, Chung KY, Pang WF, Leung CB, et al. Arterial pulse wave velocity and peritoneal transport characteristics independently predict hospitalization in Chinese peritoneal dialysis patients. Perit Dial Int. 2010 Jan-Feb;30(1):80-5.

28 Van Biesen W, Williams JD, Covic AC, Fan S, Claes K, Lichodziejewska-Niemierko M, et al.; EuroBCM Study Group. Fluid status in peritoneal dialysis patients: the European Body Composition Monitoring (EuroBCM) study cohort. PLoS One. 2011 Feb;6(2):e17148.

29 Vongsanim S, Davenport A. Factors associated with systolic hypertension in peritoneal dialysis patients. Nephrol. DOI: 10.1007/s40620-019-00633-y.

30 McCafferty K, Fan S, Davenport A. Extracellular volume expansion, measured by multifrequency bioimpedance, does not help preserve residual renal function in peritoneal dialysis patients. Kidney Int. 2014 Jan;85(1): 151-7.

31 Davenport A. Effect of intra-abdominal dialysate on bioimpedance-derived fluid volume status and body composition measurements in peritoneal dialysis patients. Perit Dial Int. 2013 Sep-Oct;33(5):578-9.

32 Tangvoraphonkchai K, Davenport A. Changes in body composition following haemodialysis as assessed by bioimpedance spectroscopy. Eur J Clin Nutr. 2017 Feb;71(2):169-72.

33 Davenport A. Changes in N-terminal pro-brain natriuretic peptide correlate with fluid volume changes assessed by bioimpedance in peritoneal dialysis patients. Am J Nephrol. 2012;36(4):371-6.

34 Holt S, Goldsmith D. Clinical Practice Guidelines. Cardiovascular Disease in CKD [accessed 2018 Jun 1]. Available from: https://renal.org/wp-content/uploads/2017/06/cardiovascular-disease-in-ckd5th-edition-1.pdf.

35 Han SH, Kang EW, Yoon SJ, Yoon HS, Lee HC, Yoo TH, et al. Combined vascular effects of HMG-CoA reductase inhibitor and angiotensin receptor blocker in non-diabetic patients undergoing peritoneal dialysis. Nephrol Dial Transplant. 2011 Nov;26(11):3722-8.

36 Lu Q, Cheng LT, Wang T, Wan J, Liao LL, Zeng J, et al. Visceral fat, arterial stiffness, and endothelial function in peritoneal dialysis patients. J Ren Nutr. 2008 Nov; 18(6):495-502.

37 Davies SJ, Woodrow G, Donovan K, Plum J, Williams P, Johansson AC, et al. Icodextrin improves the fluid status of peritoneal dialysis patients: results of a double-blind randomized controlled trial. J Am Soc Nephrol. 2003 Sep;14(9):2338-44.

38 Krzanowski M, Janda K, Dumnicka P, Dubiel M, Stompór M, Kuśnierz-Cabala B, et al. Relationship between aortic pulse wave velocity, selected proinflammatory cytokines, and vascular calcification parameters in peritoneal dialysis patients. J Hypertens. 2014 Jan;32(1):142-8.

39 Yong K, Ooi EM, Dogra G, Mannion M, Boudville N, Chan D, et al. Elevated interleukin-12 and interleukin-18 in chronic kidney disease are not associated with arterial stiffness. Cytokine. 2013 Oct;64(1):39-42.

40 Davies SJ, Davenport A. The role of bioimpedance and biomarkers in helping to aid clinical decision-making of volume assessments in dialysis patients. Kidney Int. 2014 Sep;86(3):489-96.

41 Mohamed A, Davenport A. Sodium loss, extracellular volume overload and hypertension in peritoneal dialysis patients treated by automated peritoneal dialysis cyclers. Int J Artif Organs. 2019 Aug 12:391398819864368. 